\title{
THE OVERSEAS GEOMETRIC ACCURACY VALIDATION OF ZY-3 SATELLITES IMAGES
}

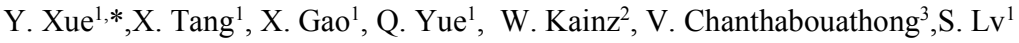 \\ ${ }^{1}$ Land Satellite Remote Sensing Application Center (LASAC), Ministry of Natural Resource (MNR), Beijing, China - (xueyc, \\ tangxm, gaoxm, yueqx, lvss)@lasac.cn \\ 2 Department of Geography and Regional Research, University of Vienna, Austria - wolfgang.kainz@univie.ac.at \\ ${ }^{3}$ National Geographic Department, Ministry of Home Affairs, Lao P.D.R. - vns.chan@gmail.com
}

\section{Commission III, WG IV/3}

\begin{abstract}
KEY WORDS: ZY-3 Satellites, China's First Civil Stereo Imaging Satellites, National Spatial Infrastructure, Geometric Accuracy
\end{abstract} Evaluation, DOM and DSM Generation

\begin{abstract}
:
With China's first stereo imaging satellite ZY-3's successful launch and two satellites networking operation, space remote sensing becomes an important means of data acquisition for survey and mapping, and geographic information updating in China.The updating period of 4D products in China is shortened by one third, the geographic information updating capability was improved more than 2 times.After more than eight years of stable and continued earth observation, ZY-3 statellites data coverage has gradually achieved more than half of the earth land which enables the overseas service for global geospatial application. Focusing on the Chinese domestic mapping applications and services, the data of ZY-3 and other domestic land observation satellites can be real-time pushed and distributed across China through the satellite cloud service platform, and worldwide for facilitating the "Belt and Road" initiative, which is promoted through the GEOSS as well for global application.
\end{abstract}

This paper presents the overseas geometric accuracy evaluation and validation of ZY-3 satellites images, which was carried out systematically for the first time abroad and complements with China's mapping evaluation practices to provide a comprehensive technical guidelines for global mapping application. The direct location accuracy of ZY-3 satellites sensor corrected image products were further evaluated and verified cooperating with the University of Vienna, Austria. The location deviations in mountain area and in flat area were analyzed and compared to check the deviation pattern and validate their consistency with the results in China.Utilizing China's ZY-3 satellites images ranging from 2012-2018, the DSM was generated automatically based on a semiglobal optimization method (Yue et al. 2016) using self-developed software SDP without ground control points but using SRTM data as elevation reference to improve the geometric accuracy. The result shows the elevation accuracy of DSM is $3.19 \mathrm{~m}(\mathrm{RMSE})$, satisfying the local requirement for $10 \mathrm{~m}$ interval contour mapping. The planar accuracy of DOM generated without ground control points utilizing ZY-3 satellites images within 2012-2015 is $2.46 \mathrm{~m}$ (RMSE). With 128 orthorectified aerial photo slots provided by NGD of Laos as reference, The planar accuracy of the DOM generated utilizing ZY-3 satellites images from $2016-2018$ is $1.65 \mathrm{~m}$ (RMSE), which can satisfy the local 1:25000 planar accuracy requirement and overlay with the local 1:2000 transportation data exactly. The overseas geometric accuracy evaluation and validation of ZY-3 satellites images provides a systematic and practical guidance for future global applications by complementing with china's experience, which would be beneficial to overseas users in acknowledging the usage of ZY-3 satellites data and help to promote it's global applications.

\section{INTRODUCTION}

With China's first stereo imaging satellite ZY-3's successful launch and two satellites networking operation, space remote sensing becomes an important means of data acquisition for survey and mapping, and geographic information updating in China.The updating period of $4 \mathrm{D}$ products in China is shortened by one third, the geographic information updating capability was improved more than 2 times.Utilizing ZY-3 satellites images, China's national $2 \mathrm{~m}$ Ground Sample Distance (GSD) Digital Orthorectified Image (DOM) and $15 \mathrm{~m} / 10 \mathrm{~m}$ grid Digital Surface Model (DSM) database has been constructed, which was updated annually and biennially to meet various needs for high precision geo-information products. ZY-3 satellites play a significant role in providing high accuracy geoinformation data for national major surveying and mapping projects. After more than eight years of stable and continued earth observation, ZY-3 satellites data coverage has gradually achieved more than half of the earth land which enables the overseas service for global geospatial application. Focusing on the domestic mapping applications and services, the data of ZY-3 and other domestic land observation satellites can be realtime pushed and distributed across China, and even worldwide through the satellite cloud service platform for facilitating the "Belt and Road" initiative, which is promoted through the GEOSS as well for global applications.

ZY-3 satellites image with three independent cameras looking at forward, nadir, and backward respectively with $2.1 \mathrm{~m}$ Ground Sample Distance (GSD) nadir images, and the interior orientation accuracy is less than one pixel after calibration. ZY302 satellite enhanced the forward and backward GSD from $3.6 \mathrm{~m}$ of ZY-3 01 to $2.6 \mathrm{~m}$ and reset the nadir and multispectral cameras to look at the same angle in order to improve image fusion quality through the optimized design. The multi-spectral camera image with a $5.8 \mathrm{~m}$ GSD and four spectral bands including blue, green, red and near infrared, the registration accuracy of multispectral bands is less than 0.15 pixels. The

*Corresponding author 
objects' spectral characteristics can be acquired simultaneously together with the terrain topography for the environmental and ecological applications, such as wetland and grassland environment monitoring (Wang et al., 2015; Zhang, 2016; Zhao et al., 2018; Kovacs et al., 2018). Moreover, Digital Surface Models (DSM) of the study area can be generated simultaneously with an accuracy, in terms of Root Mean Square Error (RMSE), 1.5-4.2 $\mathrm{m}$ in flat area, 3.9-6.9 $\mathrm{m}$ in urban area, and $5.7-8.2 \mathrm{~m}$ in mountain area, which proves to be better than Shuttle Radar Topography Mission (SRTM) (Fratarcangeli et al., 2016). The DSM generated from triplet view images ensure the stable high accuracy and good quality especially in mountainous areas (Xue et al., 2015) through the triplet imaging advantage of ZY-3 satellites to efficiently eliminate the blunders with more detail compared with two view images especially in steep terrain area, which is important for terrain correction of the multi-spectral images and can effectively improve the accuracy of biomass estimation (Gao et al., 2014; Yang et al., 2015).

Through the multi-constrained block adjustment without ground control points, the planner accuracy of ZY-3 satellites images can be better than 10m(RMSE), and the vertical accuracy can be better than 5m(RMSE)(Angelo, 2013; Tang et al., 2015; Gong et al., 2017; Wang et al., 2017).The planar accuracy of ZY-3 satellites image products can be further improved to within $3 \mathrm{~m}$ (RMSE), and the elevation accuracy can be within $2 \mathrm{~m}$ (RMSE) with a few ground control points introduced to meet the requirements of China's 1:50,000 or even larger scale like 1:25,000 mapping with the performance even better than SPOT, ALOS et.al(Gong et al., 2017; Liu et al., 2012; Pan et al., 2013; Tang et al., 2015; Toutin, Cheng, 2003; Wang et al., 2014; Wang et al., 2017; Xu et al., 2017; Zhang et al., 2014). The vertical accuracy of ZY-3 satellites stereo images, especially in the plain area, can be significantly improved using ICESat/GLAS, ZY-3 02 laser altimeter and SRTM etc data as elevation reference.

This paper presents the overseas geometric accuracy evaluation and validation for ZY-3 satellites images. The first overseas systematic geometric accuracy evaluation and validation of ZY-3 satellites images was conducted through cooperating with the University of Vienna, Austria and National Geographic Department (NGD), Ministry of Home Affairs of Lao PDR., which was carried out with the accuracy evaluation of primary location and DSM/DOM generation separately abroad and complements with China's mapping evaluation results to provide a comprehensive technical guidelines for global mapping applications.

\section{OVERSEAS GEOMETRIC ACCURACY EVALUATION OF ZY-3 SATELLITES IMAGES}

\subsection{Primary Location Accuracy Evaluation of ZY-3 Satellites Images in Austria}

In order to further verify the overseas location accuracy of ZY3 satellites sensor corrected image products, the University of Vienna, Austria, uses the open-government 30 centimeter resolution aerial images as the checking data, selects dense and evenly distributed total 1,1670 checkpoints in 100 square kilometers mountains area shown in Region of Interest (ROI) A and 2,600 checkpoints in 80 square kilometers flat area shown in ROI B respectively to evaluate the primary location accuracy of ZY-3 nadir image.One scene of ZY-3 02 satellite nadir image in August 8, 2016 covering the test sites was chosen for image direct location accuracy evaluation. The two sites location in the scene of ZY-3 nadir image is shown in Figure 1.

The location deviations distribution and the normalized histogram of the mountain and flat test sites are shown in Figure 2 and 3 separately.Comparing the deviations distribution of two sites, we can see that the deviation pattern in $\mathrm{x}$ direction is different. Comparing with each other, the most deviations in mountain area show negative with higher magnitude while positive in flat area. While both the deviations in $\mathrm{y}$ direction show negative with different magnitude indicating possible systematic deviations which can be further excluded through further block adjustment processing for the improvement of images' location accuracy.The deviations of two sites are shown against with the images in Figure 4, the obvious systematic deviations tendency was observed. From the normalized histogram of the deviations in two sites shown in Figure 3, we can see that most deviations in mountain area are higher with the median value of about $25 \mathrm{~m}$ than those in flat area, with the median value of about $15 \mathrm{~m}$, the overall situation is consistent with most of the results in China (Zhang, 2014; Tang et al., 2015; Gong et al., 2017).

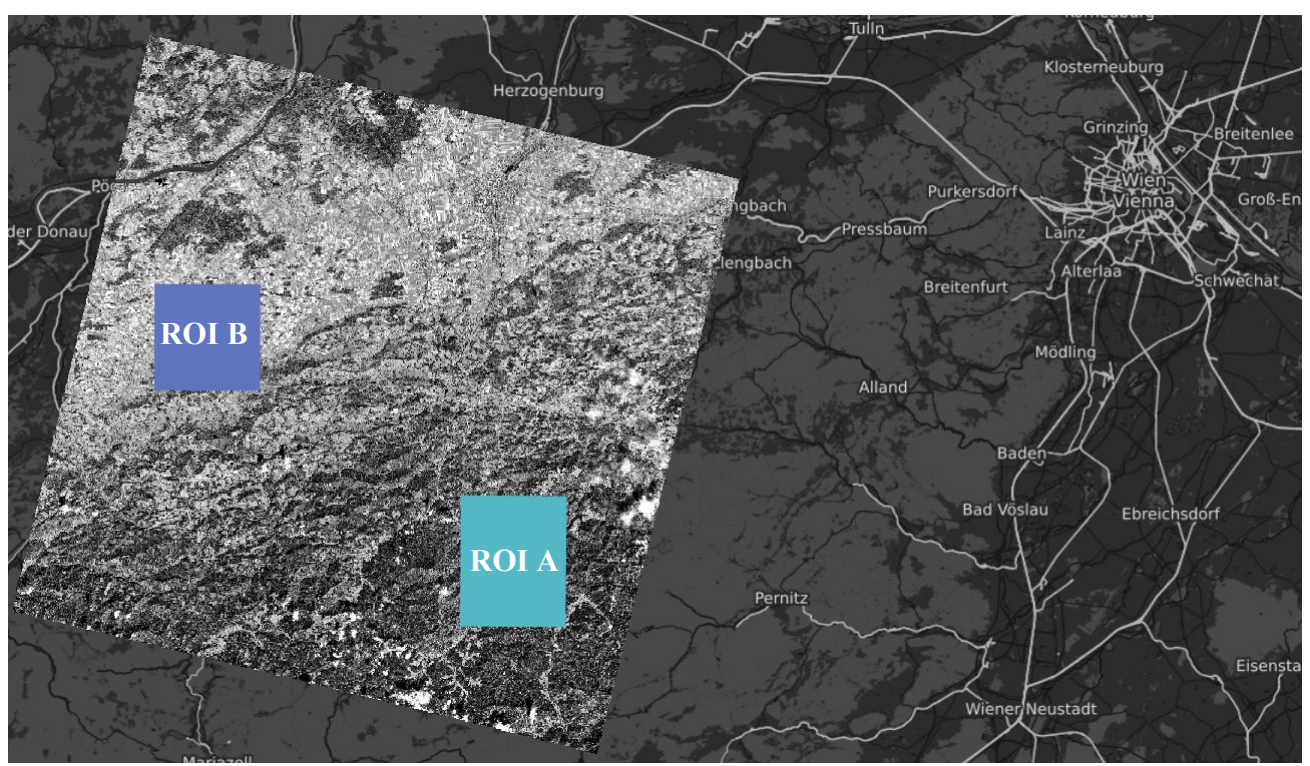

Figure 1. The two sites for image direct location accuracy evaluation in Austria 


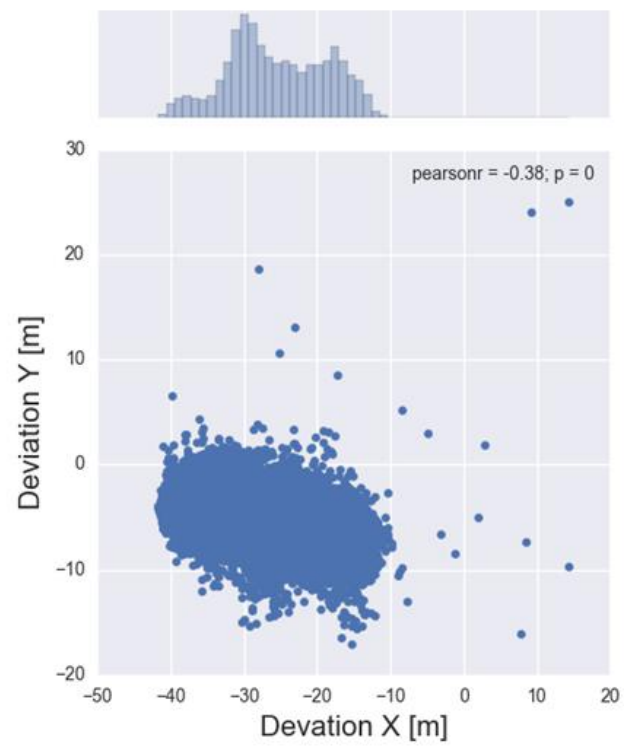

(a) The mountain area $\mathrm{A}$

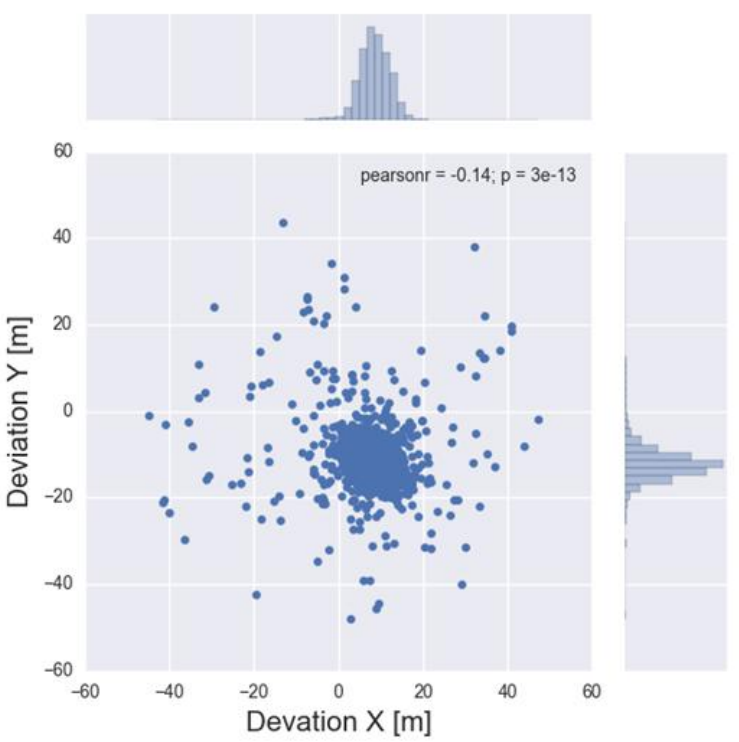

(b) The flat area B

Figure 2. The deviations pattern of checking points

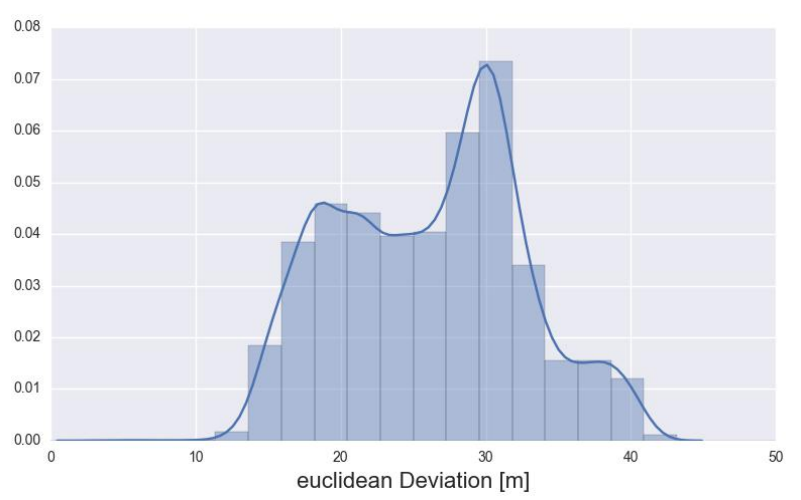

(a) The mountain area $\mathrm{A}$

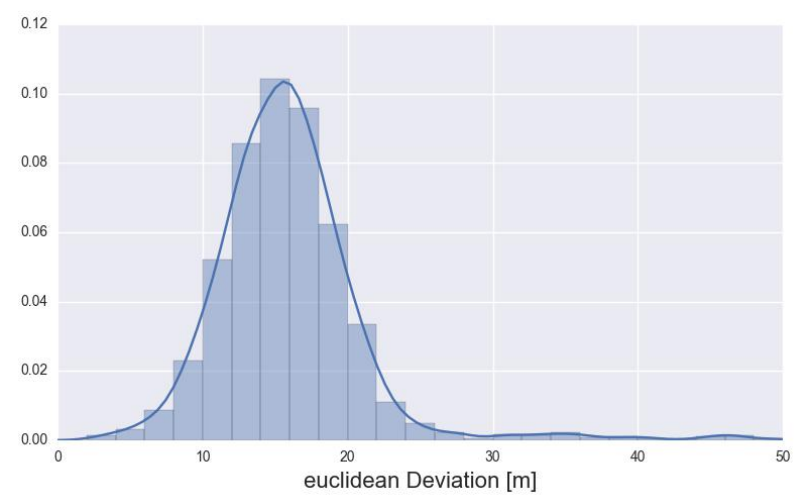

(b) The flat area B

Figure 3. The normalized histograms of the euclidean deviations of checking points

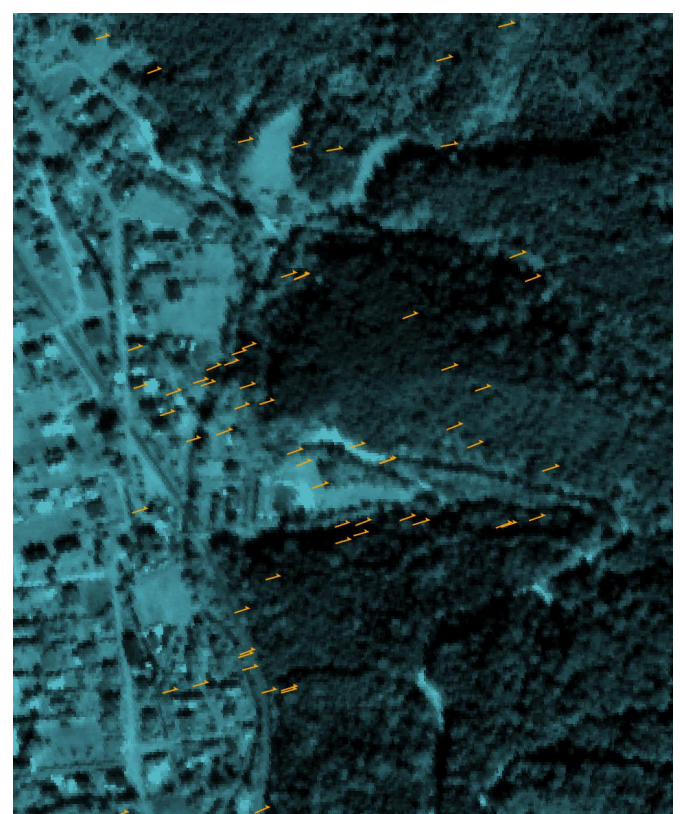

(a) The mountain area $\mathrm{A}$

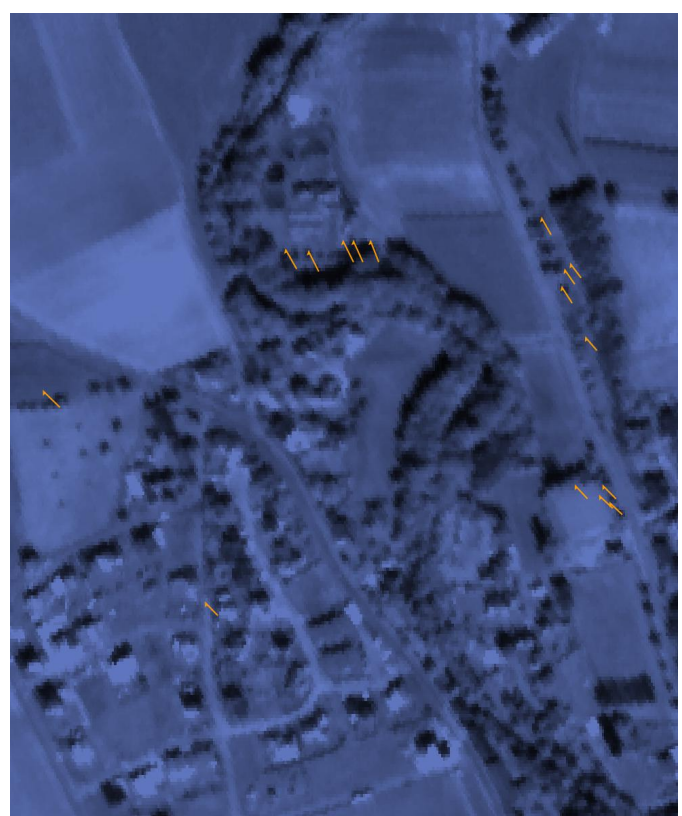

(b) The flat area B

Figure 4. The regional distribution of the deviations of checking points 


\subsection{Geometric Accuracy Evaluation of ZY-3 Satellites Images for DSM and DOM Generation in Lao PDR}

In order to systematically evaluate the overseas mapping potential of ZY-3 satellites data, the DSM and DOM were generated covering most territory of Laos and the geometric accuracy was validated through collaborating with National Geographic Department (NGD), Ministry of Home Affairs of Lao P. D. R.The study area covers about 230,000 square kilometers in Laos, which is 1,050 kilometers long from north to south, 500 kilometers wide from east to west and only 105 kilometers wide in the narrowest area. The overall terrain is high in the north and low in the south. The north is neighbored with the western plateau in Yunnan, China. The steep mountain rises above the plateau at the highest altitude of 2820 meters. The plateau is 2,000-2,800 meters high above the sea level. The terrain altitude declines from the north to the south of Vientiane. The eastern part is the Changshan Mountain on the border of Laos and Vietnam, which inclines toward the Mekong Valley and forms a plateau, while the western part is the plain area along the Mekong River. Mountainous and plateau areas account for $80 \%$ of the country's whole territory, and the national forest coverage rate is around $50 \%$.

Due to the limited budgets and human resources, the first version of local 1:50,000 base maps were almost finished by NGD with the help of foreign countries. For large scale mapping, only $0.5 \mathrm{~m}$ resolution digital aerial photo covering Laos 92\% territory was available for map production, which was acquired in different years, that is 2011 for southern part, 2013 for middle part and 2015 for northern part separately. Due to the fast urban development and intense economic activities, there is a pressing need to update the geoinformation data for local sustainable planning and development.

\subsubsection{Methodology of DSM and DOM Generation}

Due to the local tropical and subtropical climate, most time is rainy season, it is difficult to get clear satellite images without clouds covering the whole territory within short time, we even can not get one clear satellite image for some area in Laos southern part during eight years of ZY-3 operation period. For DSM and DOM generation in difficult optical imaging area, the multi-temporal images even with large area clouds covering were used to generate one whole DSM without blunder holes and clear DOM through automatic clouds detection and repatching using corresponding tie points matching technique of stereo images based on photogrametric methodology to ensure the geometric accuracy within multi-temporal images in order to improve the utilization efficiency of the satellites images for mapping production. The overall workflow is shown is Figure 5.

Cloud image patching is implemented by replacing images with thick cloud cover with overlapped images without cloud cover. The premise of this substitution method for removing clouds is fulfilled through the high precision geometric registration between the image to be patched and the clear substitute image, it is possible to ensure that the geometrical accuracy of the repaired image will not be significantly decreased as much as possible, which can be used for surveying and mapping production. This research will use the Rational Function Model of ZY-3 satellites images to establish the relationship of the geometric transformation between the image to be repaired and the substitute image. Extracting image corresponding points in the overlay area, performing block adjustment to make the image corresponding point intersect at the same ground point to establish the correspondence relationship between the image points to be patched - ground points of DSM - the substitute image points, and extracting the DSM with the clear substitute stereo images to realize image patching. A method of automatic integrated processing of multi-temporal satellite images with thick clouds for DSM and DOM generation is proposed for this research, through the cloudy images area detection and the precise location of clear image in the object and image space to realize thick clouds automatic detection and patching, this method can realize seamless mosaic between the processing images.

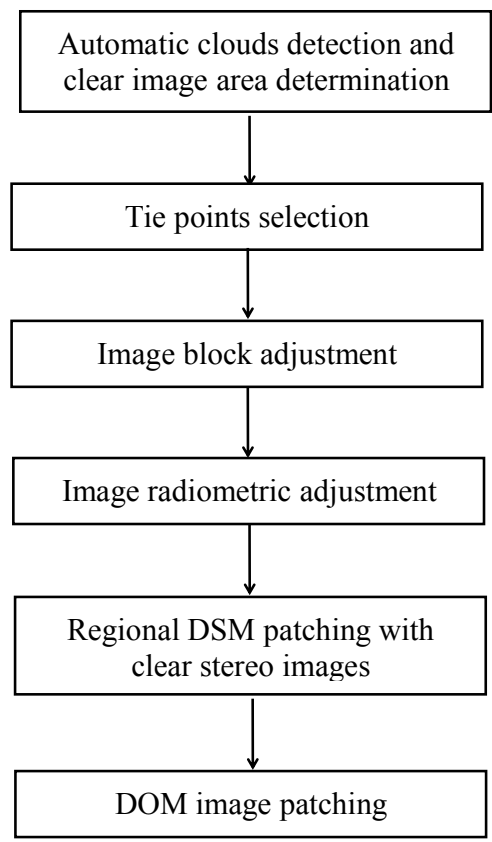

Figure 5. The workflow of automatic DSM and DOM generation with multi-temporal images for clouds removal

\subsubsection{Geometric Accuracy Evaluation}

The DSM was generated automatically based on the proposed method using a semi-global optimization strategy (Yue et al., 2016) without ground control points but using SRTM data as elevation reference to improve the elevation accuracy.The DSM with $10 \mathrm{~m}$ grid generated using self-developed software SDP utilizing China's ZY-3 satellites images ranging from 20122018 in order to cover most of the Laos territory is shown in Figure 6. The most area of the overall DSM shows quite smooth with terrain detail in $10 \mathrm{~m}$ grid using the proposed multitemporal images patching technique.

In order to systematically evaluate the geometric accuracy of the Laos DSM generated from ZY-3 satellites images, the dense and evenly distributed 131 GPS checking points provided by NGD were used to validate the elevation accuracy. The result is shown in Table 1, the vertical accuracy of the DSM is 3.19 $\mathrm{m}(\mathrm{RMSE})$ as shown in the table, and the maximum is within $10 \mathrm{~m}$, which proves the stable consistency with most of the practices in China and satisfy the local elevation requirement for $10 \mathrm{~m}$ interval contour mapping in Laos and 1:50,000 scale mapping in China. 


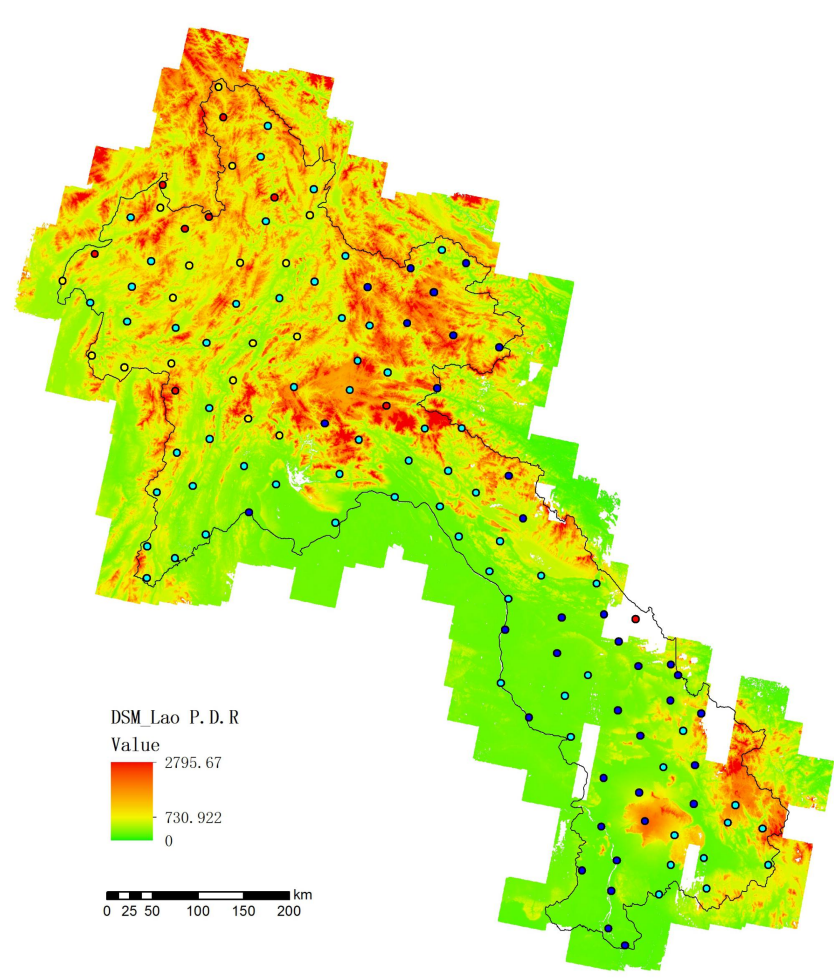

Figure 6. The DSM generated utilizing ZY-3 satellites images in 2012-2018 and the distribution of 131 GPS checking points

\begin{tabular}{|c|c|c|c|}
\hline No of CPs & RMSE(m) & $\operatorname{MaxEr}(\mathrm{m})$ & $\operatorname{MinEr}(\mathrm{m})$ \\
\hline 131 & 3.19 & 7.47 & -8.56 \\
\hline
\end{tabular}

Table 1.The elevation accuracy of DSM

The DOMs were generated automatically using ZY-3 satellites images ranging from 2012-2015 without GCPs and 2016-2018 with aerial photo plots as references to provide a new version for Laos mapping application. The two versions were compared to check the variation in planar accuracy. The DOM generated without ground control points utilizing ZY-3 satellites images within 2012-2015 is shown in Figure 7. In general we can see the image in most of the study area is clear utilizing the proposed DOM generation methodology. The color balance of the DOM in the right down area is not so good and manual adjustment need to be fulfilled in order to improve it as a whole for map production.

The planar accuracy of DOM generated without ground control points within $2012-2015$ is $2.46 \mathrm{~m}$ using $1280.5 \mathrm{~m}$ GSD orthorectified aerial photo plots provided by NGD as checking data.The statistics are shown in Table 2 with the two version planar accuracy comparison. The deviations distribution is shown in Figure 8. We can see that there still exits systematic errors need to be further excluded for accuracy improvement. With 128 orthorectified aerial photo slots provided by NGD of Laos as planar reference, the DOM was generated utilizing ZY3 satellites images from 2016-2018. The planar accuracy is improved to $1.65 \mathrm{~m}$ (RMSE) as listed in Table 2, which can satisfy the local 1:25000 planar accuracy requirement.The image can overlay with the local 1:2000 transportation line graph data exactly shown in Figure 9.

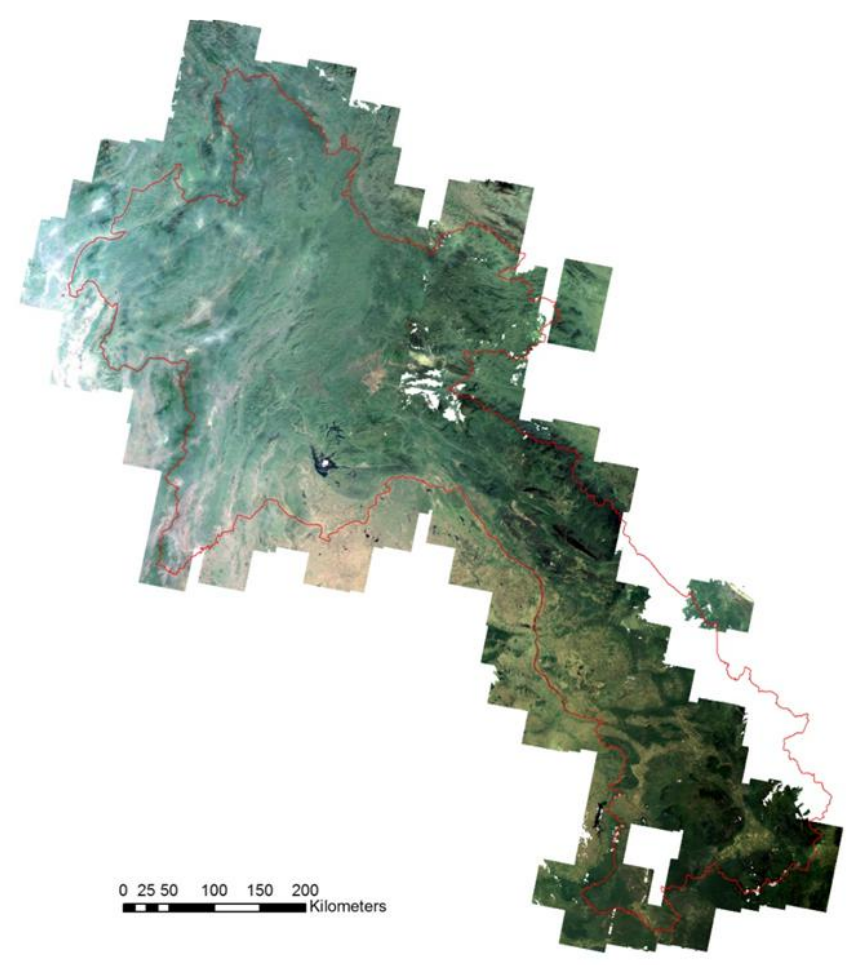

Figure 7. The DOM mosaic generated utilizing ZY-3 satellites images from 2012-2015

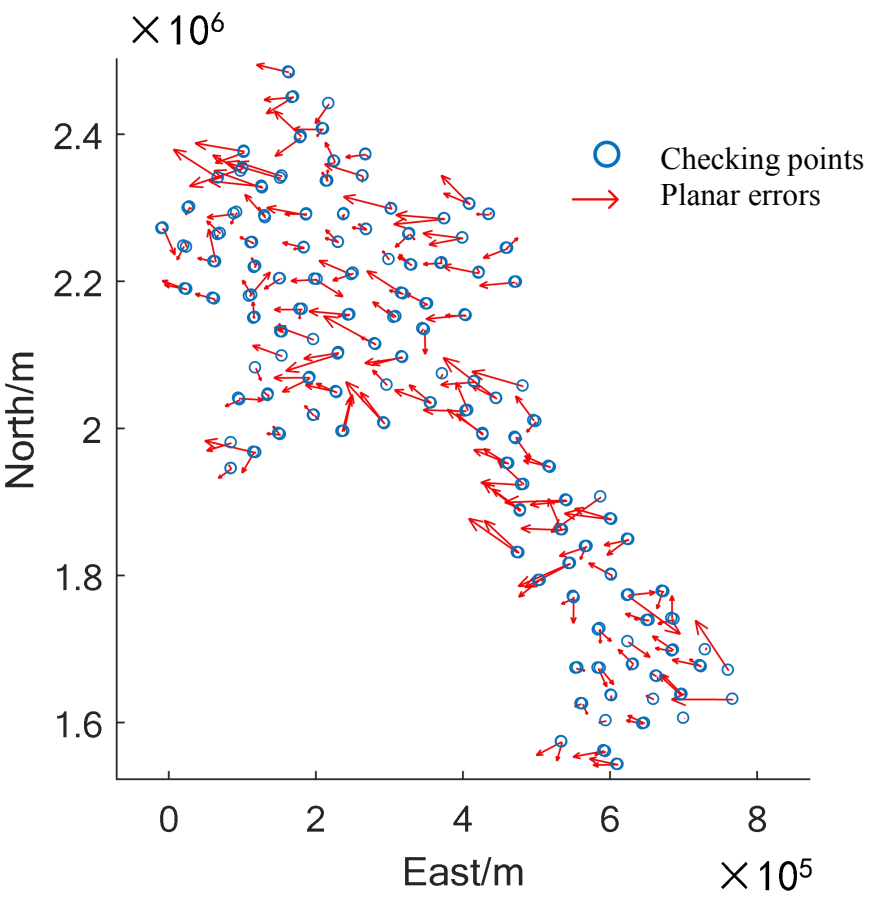

Figure 8 . The planar errors of the 223 checking points

\begin{tabular}{|c|c|c|c|c|}
\hline DOM Version & No of CPs & RMSE_X(m) & RMSE_Y(m) & RMSE_XY(m) \\
\hline $2012-2015$ & 223 & 1.89 & 1.57 & 2.46 \\
\hline $2016-2018$ & 226 & 1.19 & 1.13 & 1.65 \\
\hline
\end{tabular}

Table 2. The planner accuracy comparison of the DOMs 


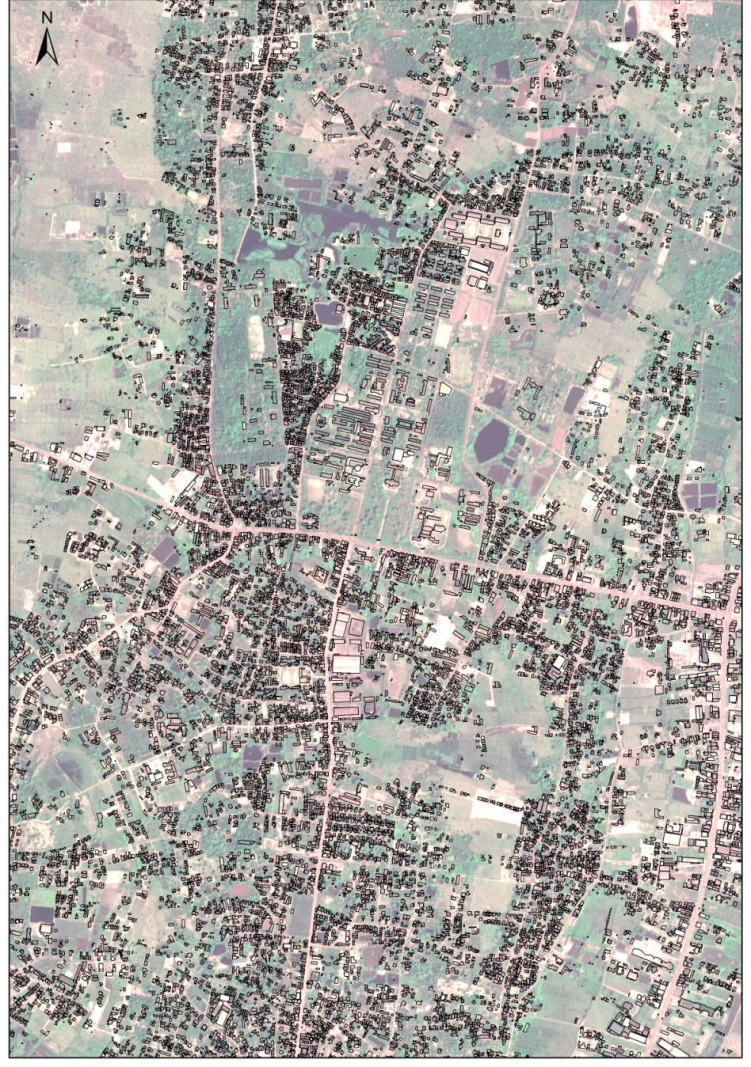

Figure 9. The DOM in Vientiane overlaid with the 1:2000 transportation line graphs provided by NGD

\section{CONCLUSION AND DISCUSSION}

Through the overseas evaluation of image's direct location accuracy of sensor corrected nadir image in Austria and the overall performance of ZY-3 satellites images for DOM and DSM generation in Laos, its potential for local 1:50,000 and 1:25,000 mapping is demonstrated and validated for global geographic information application. The evaluation and validation results show stable consistency with China's mapping practices. In general, the images overall quality is good or even better than most of the optical satellites with similar configurations (Li,Wang, 2012; Jiang et.al, 2014; Li et.al, 2014; Xu et.al, 2012).

In order to improve the map production of optical satellites for difficult imaging area with large thick clouds like Laos,the proposed DSM/DOM generation method with automatic patching can be used utilizing multi-temporal satellites images based on photogrammetric stereo images matching to ensure the geometric accuracy of DOM and DSM products. For DSM generation, the multi-view stereo images of ZY-3 satellites have technical advantages to achieve high accuracy especially for the highly varied terrain of mountainous area like Laos.Taking SRTM as a elevation constraint without ground control points, the elevation accuracy of DSM generated from ZY-3 satellites stereo images can be effectively improved to meet with the requirements of the elevation accuracy of local mapping applications.

Using laser altimeter data of satellites such as ICESat/GLAS, ZY-3 02 and GF-7, the elevation measurement accuracy of ZY3 satellites stereo images can be effectively improved, satisfying the need for high precision mapping applications outside China. ZY-03 03 satellite would carry laser altimeter to improve ZY-3 images' geometric performance automatically without GCPs which will become one important research question for the overseas applications. ZY-3 satellites stereo images can be used to timely obtain 3D terrain information of the research area for the high precision terrain correction of the multi-spectral images which can effectively improve the accuracy of biomass estimation,which acknowledged the potential for more precise applications in natural resource monitoring and management.

The overseas service nodes of the satellite remote sensing cloud service platform for ZY-3 and other Chinese domestic highresolution satellites data distribution, have been expanded in 17 countries outside China in order to promote China's satellites remote sensing technique, data and geographic information services for gradually strengthening practical cooperation along the Belt and Road region (for the website please refer to: http://sasclouds.com). Through the satellite based geographic information technology cooperation within the BRICs and GEO, we can contribute to advance the implementation of the GEOSS ten-year implementation plan and promoting the international application and service of ZY-3 satellites. With these efforts, more practices on ZY-3 images performance evaluation and validation can be carried out in the future to guide and consolidate the global applications.

\section{ACKNOWLEDGEMENTS}

This research was funded by the National Key R\&D Program of China for Strategic International Cooperation in Science and Technology Innovation (Grant No. 2016YFE0205300), the National Surveying and Mapping Scientific and Technological Fund (Grant No.2018KJ0302) and National S\&T International Cooperation Special Fund (Grant No. 2014DFA21620). We thank Dr.Michael Holzapfel, Department of Geography and Regional Research, University of Vienna, Austria for the data processing for the accuracy evaluation of ZY-3 image in Austria. We also thank the anonymous reviewers for their constructive comments and suggestions on this paper.

\section{REFERENCES}

Angelo, P D., 2013: Evaluation of ZY-3 for DSM and orthoimage generation. ISPRS - International Archives of the Photogrammetry, Remote Sensing and Spatial Information Sciences, XL-1/W1, 57-61.

Angelo, P D and Reinartz, P., 2012: DSM based orientation of large stereo satellite image blocks. Int. Arch. Photogramm., Remote Sens. Spatial Inf. Sci., vol. 39, no. B1,209-214.

Bouillon, A. et al. 2006: SPOT 5 HRS geometric performances: Using block adjustment as a key issue to improve quality of DEM generation. ISPRS J. Photogramm. Remote Sens., vol. 60, no. 3, 134-146.

Kovacs, E., Roelfsema, C., Lyons, M., Zhao,S.H.\& Phinn,S.,2018: Seagrass habitat mapping: How do landsat 8 oli, sentinel-2, zy-3a, and worldview-3 perform? Remote Sensing Letters, 9:7, 686-695 doi: 10.1080/2150704X.2018.1468101 Fratarcangeli, F., Murchio,G. , Rita,M. Di, Nascetti, A. and Capaldo,P., 2016:Digital surface models from ziyuan-3 triplet: Performance evaluation and accuracy assessment. International Journal of Remote Sensing 37 (15): 3505-3531. doi:10.1080/01431161.2016.1192308

Gao, M. L., Zhao, W. J., Gong, Z. N., Gong, H. L., Chen, Z., and Tang, X. M., 2014: Topographic correction of ZY-3 satellite images and its effects on estimation of shrub leaf biomass in mountainous areas. Remote Sensing, 6(4), 31413151. 
Grodecki, J., and Dial, G.,2003:Block adjustment of highresolution satellite images described by rational polynomials. Photogrammetric Engineering \& Remote Sensing,69(1):59-68. Jiang. Y. H., Zhang, G., Tang, X. M., Li, D. R., Huang, W. C., Pan, H. B.,2014: Geometric calibration and accuracy assessment of Ziyuan-3 multispectral images. IEEE Transactions on Geoscience and Remote Sensing,52(7):41614172.

Li, D. R., 2012: China's first civilian three-line-array stereo mapping satellite:ZY-3. Acta Geodaetica et Cartographica Sinica, 41(3):317-322.

Li, D.R. and Wang, M. ,2012: On-orbit geometric calibration and accuracy assessment of ZY-3. Spacecraft Recovery \& Remote Sensing, 33(3):1-6.

Wang, M., Yang, B., Li, D. R., Gong, J.Y., Pi, Y. D., 2017:Technologies and applications of block adjustment without control for ZY-3 images covering china. Geomatics and Information Science of Wuhan University, 42(4):427-433.

Gong, J. Y., Wang, M., Yang, B., 2017: High-precision geometric processing theory and method of high resolution optical remote sensing satellite imagery without GCP. Acta Geodaetica et Cartographica Sinica,46(10):1255-1261.

Li, G. Y., Tang, X. M., Gao, X. M., Wang, H. B. and Wang, Y. ,2016: ZY-3 block adjustment supported by GLAS laser altimetry data. The Photogrammetric Record, 31(153): 88-107. Li, L., Luo, H., Zhu, H., 2014: Estimation of the Image Interpretability of ZY-3 Sensor Corrected Panchromatic Nadir Data. Remote Sens. 6, 4409-4429.

Liu, B., Sun, X., Di, K., et al., 2012:Accuracy analysis and validation of ZY-3's sensor corrected products. Remote Sensing for Land \& Resources, (4): 36-40.

Ma, Z. L., Song, W., Deng, J. P., Wang, J. L., Cui, C. 2018: A rational function model based geo-positioning method for satellite images without using ground control points. Remote Sensing. 10. 182.

Pan, H. B., Zhang, G., Tang, X. M., et al. ,2013:The geometrical model of sensor corrected products for ZY-3 satellite. Acta Geodaetica et Cartographica Sinica,42(4):516-522.

Poli, D. A., 2007:Rigorous model for spaceborne linear array sensors. Photogrammetric Engineering \& Remote Sensing,73(2): 187-196.

Reinartz, P., Müller, R., Lehner, M., and Schroeder, M., 2006: Accuracy analysis for DSM and orthoimages derived from SPOT HRS stereo data using direct georeferencing, ISPRS J. Photogramm. Remote Sens., vol. 60, no. 3, 160-169.

Teo, T. A.,2011:Bias compensation in a rigorous sensor model and rational function model for high-resolution satellite images. Photogrammetric Engineering \& Remote Sensing,77(12):12111220.

Toutin, T.,1995:Multisource data fusion with an integrated and unified geometric modeling. EARSeL - Advances in Remote Sensing,4(2):118-129.

Toutin, T. and Cheng, P., 2003:Comparison of automated digital elevation model extraction results using along-track ASTER and across-track SPOT stereoimages. Opt. Eng., vol. 41, no. 9, 2102-2106.

Toutin, T., 2006: Generation of DSMs from SPOT-5 in-track HRS and acrosstrack HRG stereo data using spatiotriangulation and autocalibration.ISPRS J. Photogramm. Remote Sens., vol. 60 , no. $3,170-181$.

Toutin, T., Schmitt, C. V., and Wang, H., 2012: Impact of no GCP on elevation extraction from WorldView stereo data. ISPRS J. Photogramm. Remote Sens., vol. 72,73-79.

Yue, Q. X., Gao, X. M., Tang, X. M., 2016: ZY-3 DSM generation method based on semi-global optimization. Geomatics and Information Science Of Wuhan Univers, 41(10):1279-1285.
Zhang, L., Zhang, J. X., Chen, X. Y., et al.,2009:Blockadjustment with SPOT-5 imagery and sparse GCPs based on RFM. Acta Geodaeticaet Cartographica Sinica,38(4):302-310. Tang, X. M., Zhang, G., Zhu, X. Y., et al.,2012:Triple lineararray imaging geometry model of Ziyuan-3 surveying satellite and its validation. Acta Geodaeticaet Cartographica Sinica, 4 1(2): $191-198$.

Tang, X. M., Zhou, P., Zhang, G., et al.,2015:Verification of ZY- 3 satellite imagery geometric accuracy without ground control points. IEEE Geoscience and Remote Sensing Letters, 12 (1 10$)$ ): $2 \begin{array}{lllllll}1 & 0 & 0 & -2 & 1 & 0 & 4\end{array}$.

Tang, X. M., Zhou, P., Zhang, G., et al. , 20015 :Geometric accuracy analysis model of the Ziyuan-3 satellite without GCPs. Photogrammetric Engineering \& Remote Sensing, 81 ( 12 ): 927 - 934 .

Wang, L., Yang, R., Tian, Q., Yang, Y., Zhou, Y., Sun, Y. et al., 2015: Comparative analysis of GF-1 WFV, ZY-3 MUX, and HJ-1 CCD sensor data for grassland monitoring applications. Remote Sensing, 7(2), 2089-2108.

Wang, M., Yang, B., Li, D. R., Gong, J. Y., Pi, Y. D., 2017:Technologies and applications of block adjustment without control for ZY-3 images covering china. Geomatics and Information Science of Wuhan University, 42(4):427-433.

Wang, T. Y., Zhang, G., Li, D. R., et al . , 20014 :Planar block adjustment and orthorectification of ZY-3 satellite images . Photogrammetric Engineering \& Remote Sensing, 8 0 ( 6$)$ ): $559-570$.

Wang, T .Y., Zhang, G., Li ,D. R., Tang, X. M., Jiang, Y. H., Pan, H. B., Zhu, X. Y., Fang, C., 2014:Geometric accuracy validation for ZY-3 satellite imagery. IEEE Geosci. Remote Sens. Lett., 11, 1168-1171.

Wang, X., 2015: Geometric accuracy improvement and verification of remote sensing image product for the ZY-3 surveying and mapping satellite. International Conference on Intelligent Earth Observing and Applications. International Society for Optics and Photonics.

Xu, K., Jiang, Y., Zhang, G., Zhang, Q., Wang, X. ,2017: Geometric potential assessment for ZY3-02 triple linear array imagery. Remote Sens. 9, 658.

Xue, Y. C., et.al., 2015:Performance evaluation of DSM extraction from ZY-3 Three-line arrays imagery, The 2015 International Workshop on Image and Data Fusion.

Xu, W., Ge, S. L., Long, X., et al.. 2012:Radiometric image quality assessment of ZY-3 TLC camera. Space Recovery and Remote Sensing, 33(3):65-74.

Yang, A., Zhong, B., Lv, W., Wu, S. and Liu, Q., 2015: Crosscalibration of GF-1/WFV over a desert site using Landsat-8/OLI imagery and ZY-3/TLC data. Remote Sensing, 7(8), 1076310787.

Yang, A., Zhong, B., Wu, S., \& Liu, Q., 2017: Evaluation on radiometric capability of chinese optical satellite sensors. Sensors, 17(1), 204.

Zhang, H., Zhang, G., Jiang, Y. H., et al,2016:A SRTM-DEM controlled ortho-rectification method for optical satellite remote sensing stereo images. Acta Geodaeticaet Cartographica Sinica, 45 (3): $326-331$.

Zhang, M., 2016:The research on the response of floating plants to water environment index in Zaozhadian wetland based on remote sensing technology. Master thesis, Beijing Capital Normal University.

Zhang, L., Zhang, J. X., Chen, X. Y., An, H. J., 2009: Blockadjustment with SPOT-5 imagery and sparse GCPs based on RFM. Acta Geodaetica et Cartographica Sinica, 38(4), pp. 302310 . 
Zhang, Y. J., Zheng, M. T., Xiong, J .X., Lu, Y. H., Xiong, X. D., 2014: On-orbit geometric calibration of ZY-3 three-line array imagery with multistrip data sets, IEEE Trans. Geosci. Remote Sens., vol. 52, no. 1, 224-234.

Zhang, Y. J., Wang, B., Zhang, Z. X., Duan, Y. S., Zhang, Y., Sun, M. W., Ji, S. P., 2014: Fully automatic generation of geoinformation products with chinese ZY-3 satellite imagery. Photogramm. Rec. 29, 383-401.

Zhang, Y. J., Zheng, M. T., Xiong, X .D., and Xiong, J. X.,2015: Multi-strips bundle block adjustment of ZY-3 satellite imagery by rigorous sensor model without ground control points. IEEE Geosci. Remote Sens. Lett., vol. 12, no. 4, 865-869.

Zhao, J., Li, J., Liu, Q., Wang, H., Chen, C., Xu, B., et al. ,2018: Comparative analysis of chinese HJ-1 CCD, GF-1 WFV and ZY-3 MUX sensor data for leaf area index estimations for maize. Remote Sensing, 10(1), 68.

Zhou, P., Tang, X. M., Cao, N., et al. , 201016 :SRTM-aided stereo image block adjustment without ground control points. Acta Geodaeticaet Cartographica Sinica, 4 5 ( $\left.\begin{array}{lllll}1 & 1\end{array}\right)$ : 131 $8-1327$.

Zhou, P., Tang, X. M., Wang, Z. M., Cao, N.,Wang, X., 2017: Vertical accuracy effect verification for satellite imagery with different GCPS. IEEE Geoscience \& Remote Sensing Letters, 99, 1-5. 